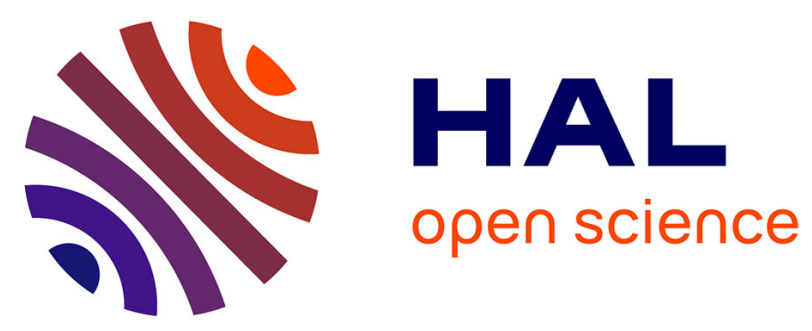

\title{
Iterative treatment with surgery and radiofrequency ablation of uveal melanoma liver metastasis: Retrospective analysis of a series of very long-term survivors
}

\author{
Vincent Servois, Toufik Bouhadiba, Sylvain Dureau, Carla da Costa, \\ Mohamed Maher Almubarak, Romain Foucher, Alexia Savignoni, Nathalie \\ Cassoux, Gaelle Pierron, Pascale Mariani
}

\section{- To cite this version:}

Vincent Servois, Toufik Bouhadiba, Sylvain Dureau, Carla da Costa, Mohamed Maher Almubarak, et al.. Iterative treatment with surgery and radiofrequency ablation of uveal melanoma liver metastasis: Retrospective analysis of a series of very long-term survivors. EJSO - European Journal of Surgical Oncology, 2019, 45, pp.1717 - 1722. 10.1016/j.ejso.2019.06.036 . hal-03487914

\author{
HAL Id: hal-03487914 \\ https://hal.science/hal-03487914
}

Submitted on 20 Dec 2021

HAL is a multi-disciplinary open access archive for the deposit and dissemination of scientific research documents, whether they are published or not. The documents may come from teaching and research institutions in France or abroad, or from public or private research centers.
L'archive ouverte pluridisciplinaire HAL, est destinée au dépôt et à la diffusion de documents scientifiques de niveau recherche, publiés ou non, émanant des établissements d'enseignement et de recherche français ou étrangers, des laboratoires publics ou privés.

\section{(ㄷ)(1) $\$$}

Distributed under a Creative Commons Attribution - NonCommerciall 4.0 International 
Version of Record: https://www.sciencedirect.com/science/article/pii/S074879831930530X

Manuscript_4462f60f194d46c56f16ba56d9aac961

Iterative treatment with surgery and radiofrequency ablation of uveal melanoma liver metastasis: retrospective analysis of a series of very long-term survivors

Vincent Servois, $\mathrm{MD}^{1^{*}}$, Toufik Bouhadiba, $\mathrm{MD}^{2}$, Sylvain Dureau, $\mathrm{PhD}^{3}$, Carla Da Costa, $\mathrm{MD}^{1}$, Mohamed Maher Almubarak, MD ${ }^{2}$, Romain Foucher, $\mathrm{MD}^{1}$, Alexia Savignoni, MD ${ }^{3}$, Nathalie Cassoux, $\mathrm{MD}, \mathrm{PhD}^{2}$, Gaelle Pierron, $\mathrm{PhD}^{4}$, Pascale Mariani, $\mathrm{MD}^{2}$.

1 Department of Radiology and Nuclear Medecine, Institut Curie, PSL Research University, 26 rue d'Ulm-75248 Paris Cedex 05

2 Department of Surgical Oncology, Institut Curie, PSL Research University, 26 rue d'Ulm-75248, Paris Cedex 05

3 Department of Biostatistics, Institut Curie, PSL Research University, 26 rue d'Ulm-75248 Paris Cedex 05

4 Department of Biopathology, Institut Curie, PSL Research University, 26 rue d'Ulm-75248 Paris Cedex 05

*Correspondence to: Vincent Servois, MD, Department of Radiology and Nuclear Medecine, Institut Curie-26 rue d'Ulm-75248 Paris Cedex 05, France

e-mail: vincent.servois@curie.fr

phone: + 331443240 00; fax: +33153104015

\section{ABSTRACT}

BACKGROUND: After treatment of primary ocular uveal melanoma (UM), up to $50 \%$ of patients will develop metastases, mostly in the liver. Systemic treatments do not provide any overall survival benefit for these patients and surgery remains the most effective therapy for selected patients. Radiofrequency ablation (RFA) alone or in combination with surgery is frequently used to spare hepatic parenchyma. When patients relapse after treatment of their first metastases, and when the liver recurrence is limited, new local liver treatment is questionable.

METHODS: A total of 14 patients with liver metastases from uveal melanoma (LMUM) were retrospectively evaluated. All patients had a complete first liver resection and a second treatment with RFA. Overall survival, recurrence-free interval after the first and the second treatment was evaluated.

RESULTS: Treatment of hepatic recurrence was percutaneous RFA for ten patients and per-operative RFA for four patients associated with new metastasectomy. The median time to onset of LMUMs 
after ocular UM treatment was 50 months, and the median time to recurrence of hepatic metastasis after the first liver treatment was 20 months. The overall survival was $70 \%$ at five years and $35 \%$ at ten years. The recurrence-free interval was $50 \%$ and $56 \%$ at two years after the first and the second treatment, respectively.

CONCLUSION: Prolonged survival can be achieved by exclusive and iterative local treatment combining surgery and RFA in a small proportion of patients with a first recurrence of isolated LMUM.

Keywords: uveal melanoma; liver metastasis; surgery; radiofrequency ablation; long survival; genomic profile 


\section{INTRODUCTION}

Uveal melanoma (UM) is the most common primary ocular malignancy among the adult Caucasian population. Despite successful treatment of the primary tumour, up to $50 \%$ of patients will develop metastases, mostly in the liver ( $90 \%$ of patients), with a metastatic median overall survival of 4 to 15 months $[1,2]$. Chemotherapy or targeted therapies do not provide any overall survival benefit for these patients [3, 4]. Indeed, UM patients have dramatically lower response rates to immunotherapy than patients with cutaneous melanomas [5-8] and other local treatments such as intra-arterial chemotherapy, chemoembolization and radioembolization have not shown a significant therapeutic efficacy. [9-13]. Intra-arterial chemosaturation with melphalan appears to be encouraging [14-16] but must be confirmed in a larger series of patients with a long follow-up. Taken together, surgery currently remains the most effective therapy for selected patients. In this setting, it has been shown that RO (microscopically complete) resection could achieve median overall survivals reaching up to 27 months compared with 11 months in patients with R1 or R2 resections [17-19]. Furthermore, it has been suggested that the use of radiofrequency ablation (RFA) alone or in combination with surgery could allow increasing the number of patients with negative margins. In our previous published series, we demonstrated that RFA ablation was as effective as surgery alone in that context [20]. When patients relapse after treatment of their first metastases, it is most often exclusively in the liver. In such situations, the management of limited recurrence remains poorly know and the relevance of a new local liver treatment is still a matter of ongoing debate. At our centre, repeated ablation of recurrent liver metastases of uveal melanoma (LMUM) has been attempted whenever technically feasible. .

Hence, the purpose of this monocentric retrospective study was to analyse the survival of patients who underwent percutaneous or per-operative RFA to treat hepatic recurrence following an initial RO surgical treatment.

\section{METHODS}

From January 2000 to December 2014, patients with LMUM who underwent hepatic resection with curative intent were identified from our prospectively maintained surgical database and retrospectively analysed. This retrospective study had IRB approval, and informed consent was waived. 
R0 resected patients were first selected. According to our institutional practice, after R0 resection, patients underwent surveillance without chemotherapy. Patients were evaluated by liver MRI every four months during a period of three years and by thoraco-abdomino-pelvic CT scan once a year. When liver recurrence was suspected a new thoraco-abdomino-pelvic CT scan was performed to rule out any extra-hepatic involvement. Patients were then discussed in a multidisciplinary oncological meeting and were offered liver local treatment combining surgery and/or RFA whenever possible. The decision was made on a case-by-case analysis taking into account the number and size of the lesions, their location (proximity to major hepatic vessels and central bile ducts), the time to onset of recurrence, the general condition of the patient and its co-morbidities.

\section{Data collection}

The patient charts were retrospectively reviewed, and the following data were collected: age, sex, characteristics of primary UM and treatment, age at time of liver metastasis and time interval from the initial treatment of the primary uveal melanoma to metastasis. For the first metastatic liver event and for hepatic recurrence, the following were recorded: intrahepatic tumour number, location and size of metastases, presence of miliary disease (more than five lesions $<5 \mathrm{~mm}$ located mainly in the area of the hepatic subcapsular region), type of surgical resection of the first metastases, type of treatment of recurrence of metastases with RFA, recurrence-free interval between the two procedures, treatment of metastatic recurrence after the second procedure, the date of last followup and date of death. The metastatic tumour size was defined as the largest diameter of the metastatic lesion. The liver resections were classified as metastasectomy, segmentectomy ( 1 or 2 segments) or major hepatectomy (3 segments or more).

\section{Genomic analysis of LMUM and ocular UM}

DNAs were obtained from snap-frozen samples according to standard procedures (proteinase K/RNAseA treatment and phenol/chloroform extraction using PLGL (Eppendorf, Hamburg, Germany), then qualified and quantified with a Nanodrop and a Qubit dsDNA BR Kit (Thermo Scientific, Wilmington USA). Up to $1 \mu \mathrm{g}$ tumour and reference DNA were labelled and cohybridised to the NimbleGen or Agilent Microarrays. The slides were washed and scanned according to the manufacturers' instructions. Images were acquired, data extracted and produced files analysed on suitable devices and softwares (For Nimblegen: GenePix 4000B, V.6.6 Software, NimbleScan V.2.5, SignalMap V.1.9 (Roche NimbleGen Inc. Madison USA); For Agilent: SureScan, CytoScan V.2.7, CytoGenomics V.2.7 (Agilent Technologies, Santa Clara, USA). The quality of aCGH was assessed on $\log 2(R)$ standard deviation, smoothing signal and sex mismatch. 
Then, patients were classified according to the status of chromosome 3 (disomy " $D$ " or monosomy " $\mathrm{M}$ ") and chromosome 8 (normal " $\mathrm{nl}$ ", or with any type of gain "g"). Four risk classifications were defined as Low Risk: “D3/8nl”, Intermediate Risk: “D3/8g” and “M3/8nl”, High Risk: “M3/8g” based on the work of Cassoux et al. [21].

\section{RFA procedure and liver MRI post-RFA ablation}

The radiofrequency procedure and liver MRI post-RFA ablation were described previously [20]. Before RFA, a fine needle aspiration biopsy (22-gauge Chiba needle; Cook Medical, Bloomington, IN, USA) was obtained to confirm the malignant nature of the lesion. Expandable electrodes were then inserted under ultrasound guidance into the centre of the lesion for the per-operative procedure or under US or CT guidance for percutaneous procedure. The efficacy of RFA was evaluated by MRI two months after the procedure. Image subtraction at all dynamic acquisition phases (T1 with injection minus T1 without injection) were used to sensitize the detection of residual contrast enhancement [22]. The treatment was considered complete when no residual contrast enhancement was present in an ablation zone with a diameter larger than the initial lesion size.

\section{Statistical analysis}

Quantitative data are reported as median and ranges, and qualitative data were presented as numbers and proportions; missing data were not taken into account. The survival curves were constructed according to the Kaplan-Meier method. Overall survival was defined as the time from metastasis diagnosis to the occurrence of death from any cause. Two recurrence-free intervals were estimated. The first one was defined as the time from the treatment of metastasis to a first recurrence. The second one was defined as the time from radiofrequency ablation to a second recurrence (patients without post-RFA recurrence were censored at the date of last news.) All analyses were performed using R software (version 3.22; http://cran.r-project.org).

\section{RESULTS}

Among 97 patients operated with curative intent for LMUM between January 2000 and December 2014, 14 (14\%) had two successive local treatments in the liver. The median follow-up of these 10 men and 4 women was 15.6 ( 4 - 19.5) years following the treatment of the primary ocular UM. The median age of onset of the primary UM was 52.5 (31-70) years. Clinical characteristics and treatment of the UM are presented in Table 1. 


\section{Characteristics and treatment of the first liver metastasis and metastatic recurrence (Table 2)}

The median age of onset of LMUM was 59.5 years (range 42-72). None of the 9 patients treated with proton beam showed ocular recurrence. The median time to onset of LMUMs after UM treatment was 50 months (range 8-155). The number of LMUMs was one in four patients, two metastases in three patients, three metastases in four patients and four metastases in three patients. The mean size of the metastases was $14.8+/-12.7 \mathrm{~mm}$. The lesions were unilobar in nine patients $(64 \%)$ and bilobar in five patients (36\%). All these patients underwent R0 resection of their lesions.

Before the recurrence of LMUM, no patients received systemic treatment after treatment of the first metastases. The median time to recurrence of hepatic metastasis was 20 months (range 2-123). Four patients $(29 \%)$ recurred within one year and the remaining ten $(71 \%)$ recurred after one year. Recurrences were single LMUMs in nine patients, two LMUMs in one patient, three LMUMs in two patients, and five LMUMs in two patients. The mean size of the all lesions was $9.4+/-6.7 \mathrm{~mm}$. A total of 11 percutaneous RFA procedures and 6 per-operative RFA procedures were performed, the locations of which are shown in Figure 1. The mean size of the lesions treated with RFA was $12.6+/$ $6.6 \mathrm{~mm}$. All patients who had a single metastasis (nine patients) had percutaneous RFA as well as one patient who had two metastases. The remaining four patients had per-operative RFA associated with new metastasectomies. In these patients, the mean size of the surgically removed lesions was $4.7+/-$ $3.4 \mathrm{~mm}$.

No complications related to surgery or radiofrequency ablation were recorded in these patients.

\section{Follow-up after treatment with RFA}

Eight patients died of new hepatic recurrences associated with pulmonary recurrence in five cases. In three patients, hepatic and pulmonary recurrences were isolated; in two patients, it was associated with bone (one patient) and cerebral involvement (one patient). All but one of these patient received chemotherapy at diagnosis of recurrence after the second treatment with RFA.

The six remaining patients were still alive at last follow-up, and four patients had received no systemic treatment. The other two patients experienced hepatic progression treated either by chemotherapy or by immunotherapy.

The overall survival after diagnosis of the first metastasis is presented in Figure 2A. Survival at 5 years was 0.70 (range $0.49-1.0$ ) and at 10 years was 0.35 (range $0.13-0.92$ ). The overall survival after post-radiofrequency ablation is presented in Figure 2B. Survival at 2 years was 0.68 (range 0.47 - 
0.99 ) and at 4 years was 0.45 (range $0.23-0.90$ ). The median follow-up after the diagnosis of the first liver metastasis was 7 (2.3-14) years. The median follow-up after the R0 resection was 3.9 (1-5.5) years.

Recurrence free survivals between the first surgical treatment of metastases and the date of onset of hepatic recurrence are provided in Figure 3A. The recurrence free survivals were 0.64 (range 0.44 0.95 ) at 1 year, 0.5 (range $0.30-0.84$ ) at 2 years, 0.36 (range $0.18-0.72$ ) at 3 years, and 0.14 (range $0.04-0.52)$ at 4 years.

Recurrence free survivals between the RFA date and the date of occurrence of a post radiofrequency metastatic recurrence are provided in Figure 3B. The recurrence was 0.71 (range $0.51-0.99$ ) at 1 year, 0.56 (range $0.34-0.90$ ) at 2 years, at 3 years 0.37 [0.18 - 0.78], and 0.19 (range $0.04-0.89$ ) at 4 years.

\section{Genomic analysis}

The genomic data of liver metastases and UM are described in Table 3. Genomic analysis of liver metastasis was contributory in $13 / 14$ patients (one technical failure). Ten patients were classified as high-risk in 5/13 (38\%), intermediate-risk in 3/13 (23\%) and low-risk in 2/13 (15\%). Three unclassified patients $(23 \%)$ presented a partial loss of $3 p(2 / 13)$ and an isodisomy of $3 p(1 / 13)$. Genomic analysis of the UM was available for $6 / 14$ patients. Only chromosome 3 status was available for one patient (FISH analysis). The five remaining patients were classified as high-risk (3/5) and low risk (2/5). Among them four patients with all genome profiling had good concordance between chromosome abnormalities of primary UM and liver metastasis.

\section{DISCUSSION}

This is a monocentric retrospective study of a small number of patients whose results should be interpreted with caution. Nevertheless, the results of this series show that radiofrequency ablation of second LMUMs recurrence after RO resection of initial LMUMs was associated with a recurrence free interval of $56 \%$ at two years and overall survivals of $70 \%$ at five years and $35 \%$ at ten years. In this setting, the present study emphasizes the benefit of RFA treatment of recurrent LMUMs in selected patients.

In the two most recent series, the number of surgically treated patients for isolated LMUMs among all the metastatic patients varies between 32 and 34\% [17-18]. Among these patients, R0 resection is 
performed in 30 to $36 \%$ of cases. The intraoperative discovery of a capsular miliary disease most often prevents RO resection. After an RO resection, in case of isolated liver recurrence, the question of a second local treatment is rarely asked and there is no established therapeutic strategy in this setting.We want thus to emphasize that this series dealt with a very highly selected population, for several reasons. First, only a small proportion of patients initially operated on with R0 resection were included in this study (14\%). Second, the onset of the first metastatic event after the treatment of the UM was late with a median delay of 50 months, a rather favourable prognostic factor according to our experience [17] and that of other authors [23]. Third, at the first metastatic event, patients had few metastases (fewer than four in 11/14 patients) without capsular miliary disease during surgical exploration. At recurrence, patients had even fewer metastases, since nine out of 14 patients had a single metastasis. For the four patients re-operated to perform RFA at recurrence, there were no cases of capsular miliary disease seen per-operatively. The recurrences were thus obviously oligometastatic, having an impact on the prognosis of these highly selected patients [23].

RFA is a well-established liver-sparing technique for the treatment of liver metastasis. We have previously shown that RFA alone or associated with surgical treatment gave the same survival results as that of surgical treatment alone for LMUM [20]. In the situation of second liver local treatment we favoured where possible the percutaneous RFA route (11/17 procedures). In addition to the usual size criteria, the percutaneous route is unfortunately not always possible given the position of metastases in the liver. We can see in Fig. 1 that among the 17 RFA procedures, nine were performed percutaneously on the anterior segments (segment $\mathrm{VI}, \mathrm{V}$ and III) versus two in the posterior segments (II and VII). This result corresponds to the most easily accessible liver segments with imaging guidance. On the other hand, the six per-operative RFA procedures were carried out in all the hepatic segments (outside segment VII) because of the better accessibility of all the lesions linked to the liver mobilization carried out by the surgeon during the laparotomy.

The definition of a long-term survival in this metastatic disease is imprecise. Some authors consider that survival greater than 12 months makes patients long-term survivors [24]. Thus, the overall survival observed in this series is particularly long for patients with LMUM: 70\% of patients alive at 5 years is an exceptional result, as is $35 \%$ of patients still alive at 10 years. Usually, patients are rarely alive at 5 years, with a median survival of 25 to 27 months [17-18]. Only our high-risk cohort [25] showed a median overall survival of 40 months in the RO operated patients, already an unusually 
long period. Data published in the literature reporting long-term surviving metastatic surgical patients are rare [19, 26]. The Hsueh series [26] focused on the analysis of 112 patients, 78 of whom had exclusive hepatic metastases. The 24 operated patients (21\%) had a 5-year survival of $39 \%$ versus $0 \%$ for non-operated patients.

In this hyper-selected population, the median survival without recurrence between the initial surgical treatment and the first recurrence was 20 months, compared to the usual 8 months [17-20]. We did not know in advance the future of these patients after the second local treatment. Interestingly, in this population, when they had a second local treatment, the new recurrence-free interval was again 2 years, the same as the first recurrence-free interval (56\% versus $50 \%$ at 2 years). Thus, we gave the patient a real second chance. In our centre, even if this attitude is not unanimously recognized, we propose systemic treatment only when the patient has a non-treatable liver disease by surgery and/or RFA. Thus, in anticipation, we always seek to favour metastasectomy to major hepatectomy during the first intervention to give priority to liver sparing in case of second local intervention.

Nevertheless, this observation implies that these are patients with slow spontaneous evolution of their disease without it being possible for us to predict this slowness of evolution on the basis of clinical or biological criteria. For UM, the metastasis risk factors are based on clinical characteristics and/or genomic analysis of the primary tumour. In the Cassoux study [21], patients were divided into three classes of metastatic risk. In multivariate Cox modelling analysis, high-risk profile (M3/8g) was more strongly associated with metastasis than were the other prognostic factors. Only a few studies [27-29] have compared the genomic profiles of the primary tumour and metastases. Our study showed that in the four cases where we could study concordance, the genomic profile was similar between the primary tumour and the metastasis. Nevertheless, once the patient's disease is metastatic, the prognostic value of the genomic profile is not established. Interestingly, in our series, $38 \%$ of patients had intermediate- or low-risk genomic profiles in metastases. However, the prognostic value of the genomic profile of LMUM should be studied on a largest series of patients.

The second recurrence was hepatic in all cases. According to our criteria, no patients have recurred at the level of RFA treated lesions. Five patients had a hepatic recurrence associated with extrahepatic metastases, appearing logical in cases of long-term survival. Ten patients with second recurrence received systemic treatment, but 4 patients with isolated hepatic recurrence had not received any systemic treatment by the time of this writing; these four patients were alive without 
new recurrence, with survivals ranging from 12 months to 39 months.

\section{CONCLUSION}

Very long-term survival can be achieved by exclusive and iterative local treatment combining surgery and RFA in a small proportion of patients with a first recurrence of isolated LMUM. Given the inefficiency of the currently available systemic treatments, we believe that this approach, which may appear aggressive, must be discussed more systematically during multidisciplinary oncological meetings at expert centres treating this pathology. Better knowledge of the biological factors explaining the evolution of this metastatic disease is nevertheless essential to improve the selection of patients who may benefit from this local therapeutic strategy.

Conflicts of interest statement: No authors have conflicts of interest to declare

\section{REFERENCES}

[1] E. Kujula, T. Makitie, T. Kivela et al., Very long-term prognosis of patients with malignant uveal melanoma. Invest Ophtalmol Vis Sci. 44 (2003)4651-9.

[2] P Rietschel, KS. Panageas, C. Hanlonet et al., Variates of survival in metastatic uveal melanoma. J Clin Oncol. 23 (2005)8076-80.

[3] JJ. Augsburger, ZM. Corrêa, AH. Shaikh et al., Effectiveness of treatments for metastatic uveal melanoma. AmJ Ophtalmol. 148 (2009)119-127.

[4] RD. Carvajal, GK. Schwartz, T. Tezel et al.,Metastatic disease from uveal melanoma: treatment options and future prospects. Br J Ophthalmol 101(2017)38-44.

[5] L. Zimmer, J. Vaubel, P. Mohr et al., Phase II DeCOG-study of ipilimumab in pretreated and treatment-naive patients with metastatic uveal melanoma. PLoS One 10(2015)e0118564(1-13).

[6] AP. Algazi, KK. Tsai, AN. Shoushtari et al., Clinical outcomes in metastatic uveal melanoma treated with PD-1 and PD-L1 antibodies. Cancer 122(2016)3344-53.

[7] LA. Kottschade, RR. McWilliams, SN. Markovicet al., The use of pembrolizumab for the treatment of metastatic uveal melanoma. Melanoma Res. 26(2016)300-3.

[8] I. Karydis, PY. Chan, M. Wheater M et al., Clinical activity and safety of Pembrolizumab in Ipilimumab pre-treated patients with uveal melanoma. Oncoimmunology. 5(2016)e1143997(1-8).

[9] S. Leyvraz, S. Piperno-Neumann, S. Suciuet al., Hepatic intra-arterial versus intravenous fotemustine in patients with liver metastases from uveal melanoma (EORTC 18021): a multicentric randomized trial. Ann Oncol. 25(2014)742-6. 
[10] KV. Sharma, JE. Gould, W. Harbour et al., Hepatic arterial chemoembolization for management of metastatic melanoma. AJR Am J Roentgenol.190 (2008)99-104.

[11] A. Klingenstein, AR. Haug, CJ Zech et al., Radioembolization as locoregional therapy of hepatic metastases in uveal melanoma patients. Cardiovasc Intervent Radiol. 36 (2013)158-65.

[12] SS. Agarwala, AM. Eggermont, S. O'Day S et al., Metastatic melanoma to the liver: a contemporary and comprehensive review of surgical, systemic, and regional therapeutic options. Cancer 120 (2014)781-9.

[13] T. Sato , Locoregional management of hepatic metastasis from primary uveal melanoma. Semin Oncol. 37 (2010)127-38.

[14] R. Olofsson,C. Cahlin, C.All-Ericsson et al., Isolated hepatic perfusion for ocular melanoma metastasis: registry data suggests a survival benefit. Ann Surg Oncol.21 (2014)466-72.

[15] MS. Hugues, J. Zager, M. Farieset al., Results of a randomized controlled multicenter phase III trial of percutaneous hepatic perfusion compared with best available care for patients with melanoma liver metastases. Ann Surg Oncol. 23 (2016)109-19.

[16] TJ. Vogl, SA.Koch, G.Lotz et al., Percutaneous isolated hepatic perfusion as a treatment for isolated hepatic metastases of uveal melanoma: patient outcome and safety in a multi-center study. Cardivasc Intervent Radiol. 40 (2017)864-72.

[17] P. Mariani, S.Piperno-neumann, V. Servois et al., Surgical management of liver metastases from uveal melanoma : 16 years' experience at the Institut Curie. Eur J Surg Oncol. 35 (2009)1192-7.

[18] D. Gomez, C. Wetherill, J.Cheong et al., The Liverpool uveal melanoma liver metastases pathway : outcome following liver resection. J Surg Oncol. 109 (2014)542-7.

[19] S. Frenkel, I.Nir, K.Hendleret al., Long term survival of uveal melanoma patients after surgery for liver metastases. Br J Ophtalmol. 93 (2009)1042-6.

[20] P. Mariani, MM.Almubarak, M.Kollen M et al., Radiofrequency ablation and surgical resection of liver metastases from uveal melanoma. Eur J Surg Oncol.42 (2016)706-12.

[21] N. Cassoux, MJ. Rodrigues, C. Plancher et al., Genome-wide profiling is a clinically relevant and affordable prognostic test in posterior uveal melanoma. Br J Ophtalmol. 98(2014)669-674.

[22] M. Lee, SE.Baek, J.Moon et al., Dynamic contrast-enhanced MRI coupled with a subtraction technique is useful for treatment response evaluation of malignant melanoma hepatic metastasis. Oncotarget 7 (2016)38513-22.

[23] S. Valpione, JC. Moser, R. Parrozzani et al., Development and external validation of a pronostic nomogram for metastatic uveal melanoma. PLoS ONE 10(2015)1-12.

[24] D. Lorenzo, M. Ochoa, JM.Piulats et al., Pronostic factors and decision tree for long-term survival in metastatic uveal melanoma. Cancer Res Treat. 2017; 
[25] S. Piperno-Neumann, V. Servois, P. Mariani et al., Prospective study of surveillance testing for metastasis in 100 high-risk uveal melanoma patients. J Fr Ophtalmol. 38 (2015)526-34.

[26] EC. Hsueh, R. Essner, LI. Foshag et al., Prolonged survival after complete resection of metastases from intraocular melanoma. Cancer 100(2004)122-29.

[27] J. Trolet, P. Hupé, I. Huonet al., Genomic profiling and indentification of high-risk uveal melanoma by array CGH analysis of primary tumors and liver metastases. IOVS 50(2009)2572-80.

[28] C. McCarthy, H. Kalirai, SL. Lake et al., Insights into alterations of liver metastases from uveal melanoma. Pigment Cell Melanoma Res. 29(2015)60-69.

[29] KG. Griewank, J. van de Nes, B. Schilling et al., Genetic and clinic-pathologic analysis of metastatic uveal melanoma. Modern Pathology 27(2014)175-183. 


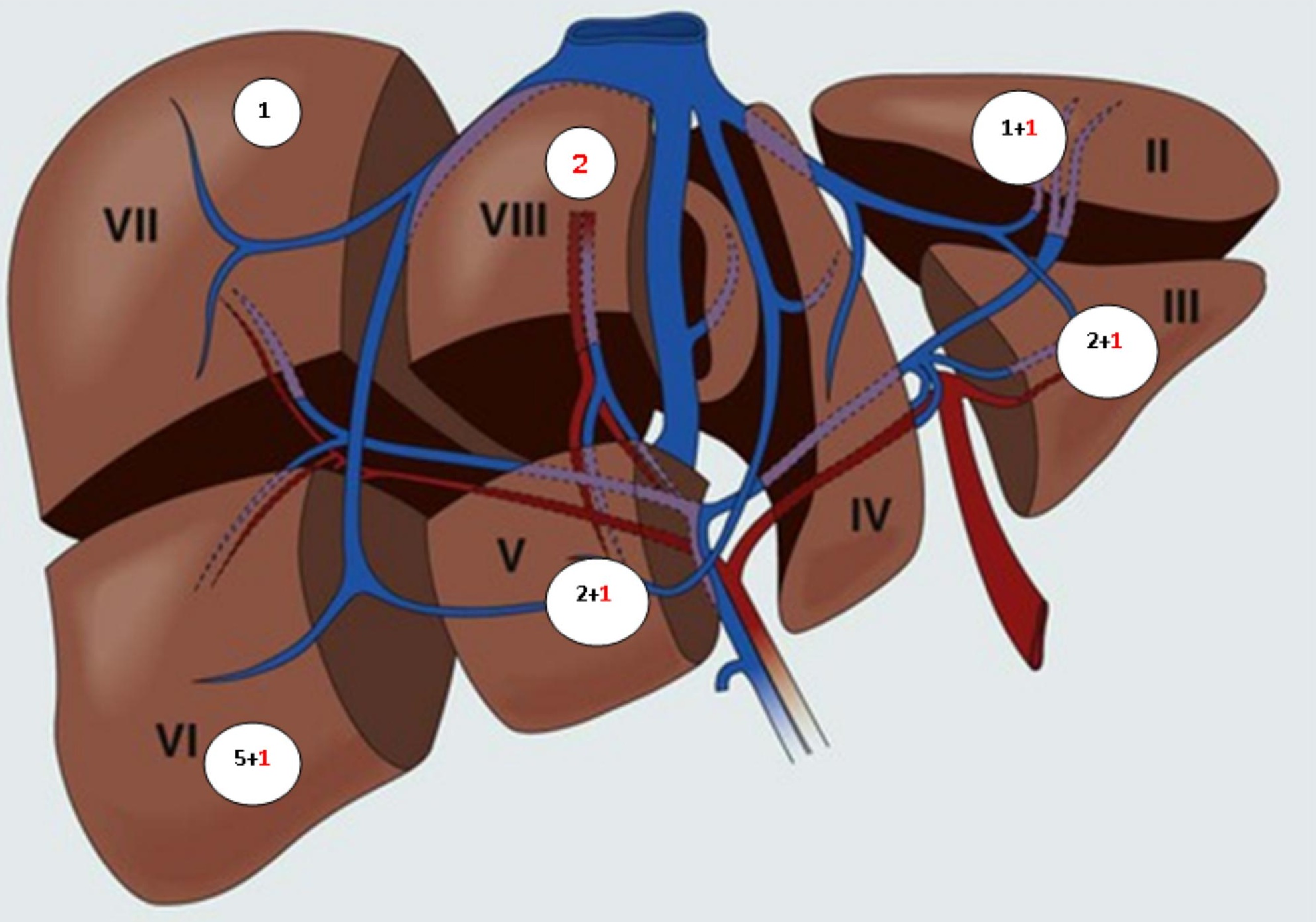




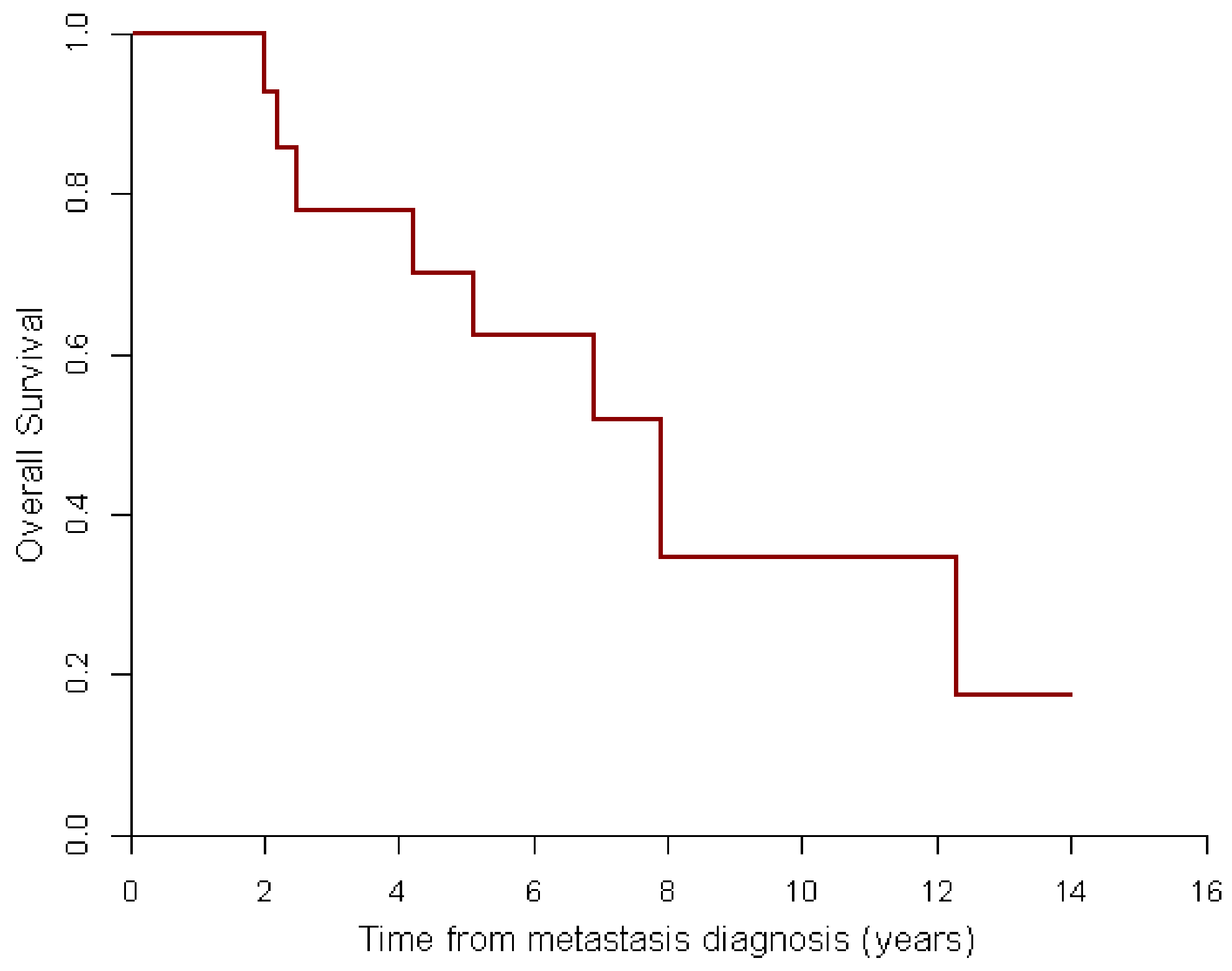

Number at risk

14

14

10

7

2

2

2

1 


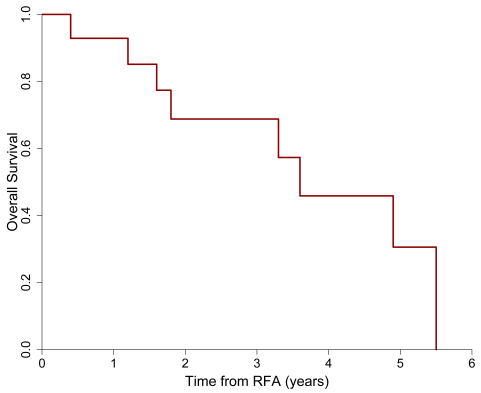

Number at risk 


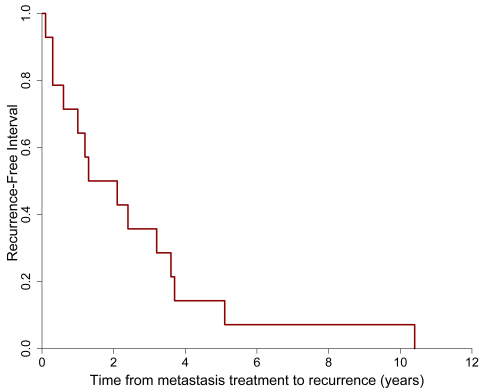

Number at risk

72




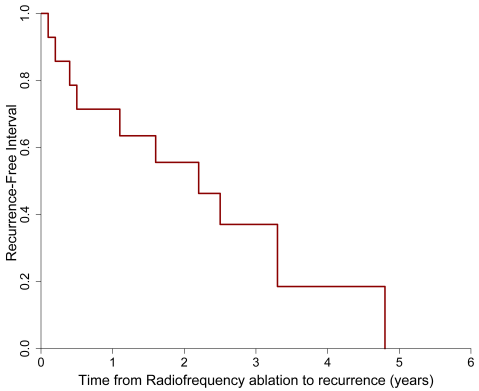

Number at risk 
Variable

Median Age

of onset metastases (years) 52.5 [31-70]

Sex ratio (male/female) M 10/ F 4

Primary Largest tumor $\quad 16.2 \mathrm{~mm}[12$ - 22]

diameter (median, $\mathrm{mm}$ )

Primary Tumor thickness $\quad 8.8 \mathrm{~mm}[3.2-14]$

(median, mm)

Treatment of primary

Proton beam radiotherapy $9(0.64)$

Enucleation $\quad 5(0.36)$

Median Age of the first liver $\quad 59.5[42-72]$

metastasis (years)

Table 1: Patient $(n=14)$ demographic and clinical features 
Median time from treatment of primary tumor

to LM (months [range])

Median time from treatment of first LM to second LM (months [range])

NA

$20[2-123]$
Size of LM treated by surgery (mean $(\mathrm{mm})$, [range]

Size of LM treated with RFA (mean $(\mathrm{mm})$, [range])

\section{Number of lesion}

1 lesion

$1<$ lesions $<4$

$\geq 4$ lesions

Topography in the liver

One lobe

$9(64 \%)$

12 (86 \%)

Bilobar

$5(36 \%)$
$4.7[1-12]$

NA

$12.6[3-26]$
6

3
9

3

2
2 (14\%)

If surgery :per-operative miliary disease *

0

0

Metastasectomy-segmentectomy

$8 / 14$

NA

Hepatectomy

$6 / 14$

NA

RFA alone

NA

$10 / 14$

RFA + metastasectomy

NA

$4 / 14$

* per-operative miliary: capsular liver disease only if patients had surgical treatment (14/14 patients for the first LM and 4/14 patients for the second LM); LM: liver metastasis

Table 2: Characteristics and treatments of the first LMUM and second LMUM 


\begin{tabular}{|c|c|c|c|c|c|c|c|}
\hline $\begin{array}{l}\text { Patient } \\
\text { number }\end{array}$ & $\begin{array}{l}\text { UM } \\
\text { Genomic }\end{array}$ & & & $\begin{array}{l}\text { LMUM } \\
\text { Genomic }\end{array}$ & & & Concordance \\
\hline & Status & Class of risk & Technic & Status & Class of risk & Technic & \\
\hline 1 & I & I & I & $\mathrm{M} 3 / 8 \mathrm{~g}$ & $\mathrm{HR}$ & Agilent & NA \\
\hline 2 & D3 & ND & FISH & $\mathrm{M} 3 / 8 \mathrm{~g}$ & $\mathrm{HR}$ & Agilent & No \\
\hline 3 & 1 & 1 & I & M3/8g & $\mathrm{HR}$ & Agilent & $\mathrm{NA}$ \\
\hline 4 & 1 & I & I & D3/8g & $\mathrm{IR}$ & Agilent & NA \\
\hline 5 & I & 1 & I & D3/8g & $\mathrm{IR}$ & Agilent & $\mathrm{NA}$ \\
\hline 6 & I & 1 & I & $3 p-/ 8 g$ & HR? & Agilent & NA \\
\hline 7 & M3/8g & $\mathrm{HR}$ & Agilent & M3/8g & $\mathrm{HR}$ & Agilent & Yes \\
\hline 8 & M3/8g & $\mathrm{HR}$ & Agilent & I & 1 & Agilent & NA \\
\hline 9 & I & I & I & $3 p-/ 8 q+$ & HR? & Agilent & $\mathrm{NA}$ \\
\hline 10 & I & I & / & $13 p / 8 g^{*}$ & HR? & Agilent & $\mathrm{NA}$ \\
\hline 11 & $\mathrm{M} 3 / 8 \mathrm{~g}$ & $\mathrm{HR}$ & Nimblegen & $\mathrm{M} 3 / 8 \mathrm{~g}$ & $\mathrm{HR}$ & Agilent & Yes \\
\hline 12 & I & I & / & D3/8g & $\mathrm{IR}$ & Agilent & NA \\
\hline 13 & D3/8nl & LR & Nimbligen & D3/8nl & LR & Agilent & Yes \\
\hline 14 & D3/8nl & LR & Agilent & D3/8nl & LR & Agilent & Yes \\
\hline
\end{tabular}

Table 3: genomic data and corresponding class of risk of primary UM ocular tumour and LMUM for the 14 patients $(M=$ =monosomy, $D=$ disomy, $g=$ gain, $n l=$ normal, $3 p$-=partial loss of short arm of chromosome 3, ${ }^{*} 13 p=$ =isodosomy of short arm of chromosome 3, 8q+=gain of long arm of chromosome $8, \mathrm{HR}=$ high risk, $\mathrm{IR}=$ intermediate risk, $\mathrm{LR}=$ low risk) 